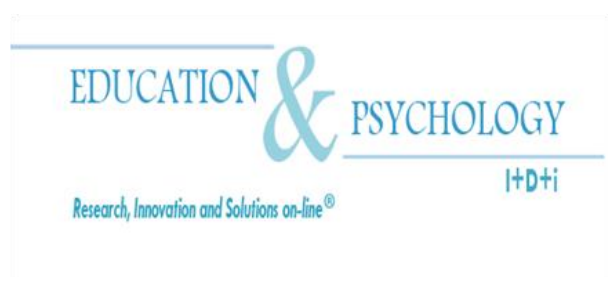

\title{
Motivaciones para la práctica físico-deportiva en adolescentes españoles, al terminar la Educación Secundaria Obligatoria
}

\section{José Macarro Moreno ${ }^{1}$, Alejandro César Martínez Baena ${ }^{1}$, Juan Torres Guerrero ${ }^{2}$}

${ }^{1}$ Facultad de Ciencias de la Actividad Física y el Deporte. Departamento de Educación Física

y Deportiva (Universidad de Granada).

${ }^{2}$ Facultad de Ciencias de la Educación. Departamento de Expresión Musical, Plástica y Corporal (Universidad de Granada).

In Memoriam del Dr. Cipriano Romero Cerezo

\section{España}

Correspondencia: Alejandro César Martínez Baena. Departamento de Educación Física y Deportiva. Facultad de Ciencias de la Actividad Física y el Deporte. Carretera de Alfacar, S/N. 18011 Granada(España).

Correo electrónico: acmartinez@ugr.es

(C) Education \& Psychology I+D+i y Editorial EOS (España) 


\section{Resumen}

Introducción. A pesar de los beneficios reconocidos sobre la práctica de la actividad físicodeportiva, las evidencias obtenidas en diversos estudios indican que no es una práctica habitual en los nuevos estilos de vida de los jóvenes, lo que supone una incidencia negativa en la calidad de vida. De ahí, el interés por conocer los principales motivos que conducirían a estos adolescentes españoles a la realización de este tipo de prácticas.

Método. Se realizó un estudio descriptivo, de corte transversal, empleando el cuestionario "Motivos de práctica físico-deportiva en la etapa adolescente", con 10 ítems que discriminan adecuadamente y obtienen una consistencia elevada. El análisis descriptivo, factorial e inferencial, nos permite obtener la opinión de 857 estudiantes acerca de los motivos que tienen y si difiere en función del sexo del tipo de centro educativo.

Resultados. Los principales motivos de participación resultan ser por estar sano y en forma, porque les gusta hacer deporte y porque les divierte para ocupar el tiempo libre. Los motivos menos valorados son para agradar a los padres y porque los familiares cercanos también practican. Respecto a las diferencias por género, los chicos conceden mayor importancia a la práctica por satisfacción con la competición, por querer hacer carrera deportiva en el futuro, por el gusto hacia el deporte y porque los mejores amigos también practican.

Discusión y conclusiones. A partir de los resultados se evidencia el interés de promover entre los adolescentes aquellos aspectos que les motivan hacia la práctica físico-deportiva.

Palabras Clave: Motivos de práctica físico-deportiva; adolescentes; género; deporte; motivación. 


\title{
Motives of Practice Physical Activity and Sport in Spanish Adolescents at the End of their Secondary Education
}

\begin{abstract}
Introduction. Despite the benefits associated to the practice of sports and physical activities, the evidences put forward in different studies show that practicing sport activities is not a habitual action in the new life style of youngsters which consequently implies a negative point in their quality of life. Here lays our interest to discover the main reasons that leads teenagers from Granada to practice sports.
\end{abstract}

Method. This is a descriptive transversal study, where we have used a questionnaire: "Reasons for practicing sports and physical activities during the teenage period". It has ten items which discriminate adequately and prove a high consistency. The descriptive, factorial e inferential analysis allows us to obtain the opinion of 857 students about the motives that have and if differs depending on the sex of the type of educational center.

Results. The main reasons to practice sports are to be healthy and fit, because they enjoy doing it and because they find it an entertaining way to spend their free time. The reasons they value less are to satisfy parents and because close relatives also practice them. Concerning genre differences, males give more relevance to practicing sports due to the satisfaction they get form competing, the idea of gaining a future in sports, the like towards sports and because their best friends also practice them.

Discussion and conclusions. From the results obtained there is an evidence of the importance of promoting among teenagers those aspects more motivating towards the practice of physical activities and sports.

Keywords: Motives for physical activity and sports; teenagers; gender; sport; motivation. 


\section{Introducción}

La actividad físico-deportiva regular es considerada como un componente esencial para una buena salud, así como para la consecución de un óptimo crecimiento y desarrollo en niños y jóvenes (Trost y Loprinzi, 2008). Diversos estudios constatan la mejora que dicha práctica produce a nivel físico, psíquico y social, así como en la calidad de vida a lo largo de los años (Moliner-Urdiales et al., 2009; Parfitt, Pavey y Rowlands, 2009). Se da una asociación directa entre la práctica sistemática y una mejor competencia percibida, alta autoestima, satisfacción con la vida y aumento de la vitalidad (Castillo y Molina-García, 2009), e inversa respecto a la presencia de depresión entre los adolescentes (Hong et al., 2009). Igualmente, dicha actividad puede ser de vital importancia en la prevención de enfermedades crónicas: cardiovasculares, diabetes, obesidad, dislipemias, osteoporosis o ciertos tipos de cáncer, entre otras patologías (Eisenmann, 2004; Lobstein, Baur y Uauy, 2004; Tounian, 2009).

A pesar de la gran cantidad de evidencias que existen sobre la importancia que puede tener la práctica habitual de la actividad física en el bienestar social y en la salud de las personas (Gálvez, Rodríguez y Velandrino, 2007) y el interés que está despertando en diversos organismos nacionales e internacionales la necesidad de promocionar la actividad físicodeportiva para un mejor estado de salud entre la población (Apovian, 2009), la encuesta de Hábitos Deportivos de los Españoles 2005 (García-Ferrando, 2005) continúa situando a España entre los países europeos con niveles más bajos de práctica deportiva.

Los niveles de práctica de actividad física y deportiva va descendiendo progresivamente con la edad (Martínez-Gómez et al., 2009a), demostrando una mayor tasa de abandono de los adolescentes (Cervelló, Escartí y Guzmán, 2007; Jurakic, Pedisic y Andrijasevic, 2009; Macarro, Romero y Torres, 2010; Prista et al., 2009; Roman, Serra-Majem, Pérez-Rodrigo, Drobnic y Segura, 2009). Diversos estudios (Thompson et al., 2009; Kijboonchoo et al., 2009) manifiestan niveles de práctica bajos al no cumplir estos grupos de edad con la pauta recomendada de 60 minutos o más de actividad físico-deportiva diaria y no haciéndose con una intensidad de moderada a vigorosa. Esto, unido a una ingesta calórica inapropiada entre los jóvenes, propicia un aumento masivo en los índices de obesidad infanto-juvenil (Woodruff, Hanning y Barr, 2009). 
La investigación de Izquierdo, Rodrigo, Majem, Roman y Aranceta (2008), sobre la obesidad en España, se detalla la situación de estos jóvenes en edad escolar con elevadas tasas de sobrepeso en la etapa prepuberal, duplicadas en los últimos 15 años. Una situación evidenciada de manera similar en países, tales como, Finlandia, Suecia o Italia (Binkin et al., 2010; Ekblom, Bak y Ekblom, 2009; Vuorela, Saha y Salo, 2009).

Con el fin de sensibilizar sobre los beneficios de adoptar hábitos de vida saludables, surge de manera reciente en España la Estrategia NAOS (Ballesteros-Arribas, Dal-re Saavedra, Pérez-Farinós y Villar-Villalba, 2007). En la línea de la Estrategia Mundial sobre Régimen Alimentario, Actividad Física y Salud (OMS, 2004), se pretende a través de una alimentación saludable y de la práctica regular de actividad físico-deportiva, una reducción significativa en la prevalencia de enfermedades crónicas. Estamos, pues, ante la necesidad de desarrollar programas de intervención que sean creados y aplicados en búsqueda de una mejora en niveles de participación activa en grupos de población joven (Judge, Petersen y Lydum, 2009; $\mathrm{Wu}, 2007$ ), aumentando la disponibilidad de recursos y oportunidades para la práctica de cualquier tipo de actividad físico-deportiva durante el tiempo libre (Yancey et al., 2009). Por tanto, deben plantearse medidas de actuación dirigidas a la promoción de salud y centradas de manera especial en materia educativa (Angelopoulos, Milionis, Grammatikaki, Moschonis y Manios, 2009). Así, la escuela es señalada como un lugar ideal para promover la actividad físico-deportiva (De Meester, van Lenthe, Spittaels, Lien y De Bourdeaudhuij, 2009; Jago et $a l .$, 2009), jugando un importante papel en la prevención de la obesidad y el sobrepeso de los más jóvenes (Coelho et al., 2008). En este sentido, a nivel nacional, destaca el Proyecto EVASYON, "Desarrollo, Aplicación y Evaluación de la Eficacia de un Programa Terapéutico para Adolescentes con Sobrepeso y Obesidad: Educación Integral Nutricional y de Actividad Física". Este programa se ha desarrollado en cinco provincias españolas (Pamplona, Madrid, Zaragoza, Santander y Granada) y está basado en el tratamiento multidisciplinar integral de adolescentes con sobrepeso y obesidad (Martínez-Gómez et al., 2009b).

La realidad indica que la actividad físico-deportiva actual no se corresponde con los nuevos estilos de vida de los jóvenes (Nuviala et al., 2009) y que resulta absolutamente necesario trabajar en el conocimiento de los principales factores demandados por estos adolescentes para que se pueda producir un aumento en sus intereses y motivaciones hacia este tipo de prácticas (Quevedo-Blasco, Quevedo-Blasco y Bermúdez, 2009; Shen, McCaughtry, Martin y Fahlman, 2009). Por tanto, precisamos conocer cuáles son las motivaciones que inducen, faci- 
litan y fortalecen la práctica de actividad físico-deportiva, con el objetivo de que los jóvenes adquieran un estilo de vida activo y lo conserven desde la adolescencia hasta la edad adulta (Kjonniksen, Anderssen y Wold, 2009).

Diversos estudios han analizado los motivos que alientan a estos jóvenes a practicar la actividad físico-deportiva, en el caso Moreno, Zomeño, Marín, Cervello y Ruiz (2009) han verificado los perfiles motivacionales según el clima motivacional, el continuo de motivación, la importancia concedida a la educación física y la asociación entre la práctica y el tiempo de actividad físico-deportiva extraescolar. Por su parte, Cecchini, Méndez y Muñiz (2002), analizaron los motivos que impulsan la práctica deportiva en la población escolar española con edades comprendidas entre los 8 y los 18 años de edad, encontrando diferencias motivacionales en función del sexo, la edad, el tipo de población y la intensidad de la motivación hacia el deporte. García-Ferrando (2005), entre la población que práctica deporte, los motivos más destacados para realizar ejercicio físico son por diversión, o por mantener y/o mejorar la salud. Moreno, Cervelló y Martínez (2007), evidenciaron que las personas mayores conceden más valor a los motivos relacionados con la salud, mientras que las más jóvenes priorizan los motivos relacionados con la apariencia. Moreno, Pavón, Gutiérrez y Sicilia, 2005, en estudiantes universitarios, encontraron en los prácticante más motivación por la competición, la capacidad personal y la aventura, mientras que los no practicantes muestran los niveles más altos de motivación en los aspectos vinculados a la salud. Martínez, Romero, Delgado y Viciana (2010), determinaron el factor motivacional de los padres y madres como influyentes en el interés de los jóvenes para la práctica de la actividad física y deportiva.

\section{Objetivos}

Existe una coincidencia de los diversos estudios encontrados de determinar los motivos que llevan a los estudiantes a practicar actividad físico-deportiva en su tiempo libre (Moreno, Gonzalez-Cutre y Chillón, 2009), con el fin de que, una vez que se conozcan, disminuir los elevados índices de abandono en la etapa adolescente (Cervelló et al., 2007). Para ello, sería necesarios la aplicación de programas de intervención educativa que ayudaran a concienciar y propiciar la práctica de la actividad física y el deporte. En esta investigación, se ha pretendido saber cuál es la realidad en el contexto de la provincia de Granada (España) sobre los motivos que llevaría a los estudiantes a la realización de este tipo de actividades. De esta forma, los objetivos son los siguientes: 
1) Construir y validar el cuestionario Motivos de práctica físico-deportiva en la etapa adolescente (MPFDA).

2) Conocer cuáles son los motivos de práctica físico-deportiva que más valoran los chicos y chicas adolescentes de Educación Secundaria Obligatoria pertenecientes a la provincia de Granada, así como la relación entre estos motivos.

3) Identificar aquellas diferencias significativas existentes en la valoración de los motivos de dicha escala entre los distintos sectores de la población analizados en función del sexo y el tipo de centro.

\section{Método}

\section{Participantes}

La población objeto de nuestro estudio está compuesta por el alumnado que estudia primer curso de bachillerato de la provincia de Granada (España). Un total de 7708 jóvenes, 3497 chicos y 4211 chicas. La muestra la conforman 857 alumnos (389 chicos -45,4 \%- y 468 chicas -54,6\%-), de 19 centros educativos diferentes. El error de la muestra que acompaña al diseño de encuesta realizado toma un nivel de confianza 1- $\alpha$ del 95.5\% $( \pm 2 \sigma)$, con error muestral de $\pm 3.2 \%$ para el conjunto de la muestra. Los centros se han seleccionado siguiendo dos criterios: abarcar con la muestra todas las zonas de la provincia de Granada y mantener en ella una proporción similar a la que existe en la población total en cuanto al tipo de centro: privado, concertado y público. Sobre la muestra inicial, 409 estudiantes (264 varones y 145 mujeres) contestaron afirmativamente su participación en prácticas físico-deportivas, en el momento de realización de la encuesta.

\section{Instrumento}

En la investigación se utiliza el cuestionario Motivos de práctica físico-deportiva en la etapa adolescente. Para la construcción de este cuestionario, se revisó la literatura existente respecto a estudios previos efectuados sobre la temática objeto de estudio y la referida a la construcción de cuestionarios (Martínez-Gómez et al., 2009a; Moreno et al., 2009; Moreno, Cervelló y Martínez, 2007; Quevedo-Blasco et al., 2009), estableciéndose una versión del cuestionario con una serie de ítems relacionados con los motivos de práctica de la actividad físico-deportiva. Tras esta primera construcción, se sometió a un juicio de diversos expertos a 
través de la utilización de la técnica Delphy, mediante el intercambio de ideas y opiniones entre integrantes de diferentes Facultades de Ciencias de la Educación y de la Actividad Física y el Deporte en materia de Actividad Física orientada a la Salud, quedando así garantizada la validez del contenido. Una vez valoradas y ajustadas la idoneidad de las preguntas, se redactó el cuestionario definitivo.

El cuestionario, que es parte de otro estudio mayor, está formado por una primera dimensión compuesta por 1 ítem con una opción dicotómica de respuesta respecto a "practica de la actividad físico-deportiva" y "no práctica de la actividad físico-deportiva" y una segunda dimensión que consta de una escala tipo Likert formada por 10 ítems referidos a "los motivos de práctica de la actividad físico deportiva", en la que tanto los chicos como las chicas valoran su grado de acuerdo/desacuerdo de cada ítem en una escala de cuatro puntos, desde "Totalmente en desacuerdo" (1) hasta "Totalmente de acuerdo" (4). Un sencillo sistema de construcción de escalas, respaldado por Morales, Urosa y Blanco (2003).

\section{Procedimiento}

La recogida de datos en esta muestra se realizó durante 3 meses, entre Marzo y Mayo de 2008. Los alumnos y alumnas participantes fueron convenientemente informados de las características del estudio, y el director de cada uno de los centros estudiados firmó un consentimiento informado para su participación. Los encuestadores siguieron un guión unificado para dar las instrucciones oportunas. Por supuesto, se les pidió que contestasen con seriedad y máxima sinceridad, asegurándoles la confidencialidad de las respuestas. El cuestionario fue administrado en horario extraescolar y se cumplimentó en aproximadamente 15 minutos.

\section{Diseño y Análisis Estadístico}

Esta investigación se realizó con un diseño descriptivo de corte transversal mediante cuestionario, con el fin de identificar los motivos en función del sexo y el tipo de centro, que llevan a los adolescentes a participar en la práctica de actividad físico-deportiva. Para los cálculos del análisis de resultados se ha utilizado el paquete estadístico SPSS v. 15.

Previamente al análisis de fiabilidad, comprobamos si los ítems discriminaban adecuadamente, calculando la correlación del ítem-total con todos los demás. Se evaluó la consis- 
tencia interna de la prueba a partir del coeficiente Alfa de Cronbach de las subescalas y en la totalidad de la prueba. Tras esto, un análisis factorial siguiendo el método de componentes principales (con rotación VARIMAX), utilizando el método de Kaiser después de la verificación de la adecuación de los datos por el test KMO y de Esfericidad de Bartlett. Dicho análisis factorial nos permite exponer aquellos componentes que se extraen de varianzas comunes a partir de una serie de medidas que se agrupan en torno a unas ideas que conformarían los factores o subescalas finales del cuestionario. Para la elección de los factores y los ítems que los conforman, se consideraron los siguientes criterios:

- Factores con un valor propio mayor que 1.

- El ítem debía presentar una saturación (carga factorial) igual a superior a 0.50.

- El ítem debía incluirse en un solo factor (aquel en el que se presentara un mayor nivel de saturación). Ítems con cargas similares en distintos factores fueron excluidos.

- Existencia de coherencia conceptual entre ítems de un mismo factor.

- Factores conformados por 3 o más ítems.

- Todos los factores debían poseer una fiabilidad por encima de 0.6.

En la primera dimensión, al tratarse de una variable nominal, se ha empleado el procedimiento de frecuencias y porcentajes. Además, para comprobar la posible relación de las variables sociodemográficas seleccionadas (sexo, centro público o concertado y tipo de centro), se ha llevado a cabo la prueba no paramétrica de Chi-cuadrado. Para la segunda dimensión, los 10 ítems que integran la escala de motivos de práctica de la actividad físicodeportiva, se ha efectuando un análisis descriptivo de cada una de los ítems (media y desviación típica), determinando cuáles son los más valorados. Para el contraste por género, teniendo en cuenta el tamaño muestral, la prueba de Kolmogorov-Smirnov presenta una distribución no normal de los datos y la prueba de Levene, varianzas desiguales, se ha optando por las técnicas no paramétricas. Para comprobar estas diferencias entre dos grupos independientes chicos-chicas, se ha aplicado la prueba no paramétrica de Mann-Whitney. Para el contraste entre los tipos de centros, se ha efectuado la prueba de varias muestras independientes de Kruskal-Wallis. En ambos casos, se ha exigido un valor de significación de $\mathrm{p}<.05$. 


\section{Resultados}

Construcción y validación de la escala

Los ítems, tal como ya se ha comentado, constituyen una definición operativa, provisional e hipotética, del comportamiento que se va a medir. Al calcular la correlación del ítemtotal con todos los demás para comprobar si estos discriminan de manera adecuada, se obtienen diversas correlaciones, oscilando entre 0.38 del ítem 1 al 0.59 del ítem 2, con una alta significación en todos los ítems en los niveles de confianza del 95\% y de $\mathrm{p} \leq .01$. Al aplicar el método de consistencia interna "alfa de Cronbach, obtuvimos un coeficiente de .81 .

Se justifica un índice KMO significativo de .79, próximo a la unidad. La prueba de esfericidad proporciona una Chi-cuadrado de 708.818 y muy alta significación $(p=.00)$, lo que nos indica la pertinencia del análisis factorial. Por tanto y para la obtención definitiva de la escala, las puntuaciones de los 10 ítems se han sometido a un análisis factorial exploratorio basado en las correlaciones mutuas entre las diversas cuestiones, las cuales pueden sintetizarse en forma de factores o ideas subyacentes de las relaciones entre los ítems. El método de extracción mediante el análisis de componentes principales con una simple rotación de normalización Varimax ha convergido en cinco iteraciones, dándonos tres componentes que explican el $65.537 \%$ de la varianza y tiene interés para la interpretación de nuestro estudio, omitiendo las cargas inferiores al valor .5

La estructura factorial muestra un factor 1 dominante, formado por 4 ítems que explica el $30.80 \%$ de la varianza, con todas las cuestiones de la escala con una carga apreciable (por encima de .5) y un índice de fiabilidad alto $(\alpha=.753)$. Un factor 2 , compuesto por 3 ítems que explica un $19.32 \%$ de la varianza, con cargas superiores a .7 y fiabilidad aceptable $(\alpha=.670)$. El factor 3, compuesto por 3 ítems que explica un $15.32 \%$ de la varianza, con una carga por encima de .7 y un índice de fiabilidad aceptable $(\alpha=.687)$. 
Tabla 1. Matriz de Componentes (con rotación VARIMAX)

\begin{tabular}{|c|c|c|c|c|}
\hline & \multirow{2}{*}{$\begin{array}{c}\text { \% Varianza } \\
\text { explicada }\end{array}$} & \multicolumn{3}{|c|}{ Factor } \\
\hline & & 1 & 2 & 3 \\
\hline ITEM5. Para encontrarme con amigos y hacer otros nuevos. & & .75 & & \\
\hline ITEM10. Porque me divierte para ocupar el tiempo libre. & & .67 & & \\
\hline ITEM7. Porque mis mejores amigos también practican. & & .66 & & \\
\hline ITEM2. Porque me gusta competir. & $30.80 \%$ & .56 & & \\
\hline ITEM9. Para agradar a mis padres. & & & .78 & \\
\hline ITEM8. Porque mis familiares cercanos también practican. & $19.32 \%$ & & .77 & \\
\hline ITEM3. Porque quiero hacer carrera deportiva. & & & .73 & \\
\hline ITEM6. Porque me gusta hacer deporte. & & & & .84 \\
\hline ITEM1. Por estar sano y en forma. & $15.32 \%$ & & & .86 \\
\hline ITEM4. Para mantener la línea y tener buen aspecto físico. & & & & .86 \\
\hline
\end{tabular}

Para determinar el tipo de relación existente entre los ítems, se ha aplicado el análisis de correlación bivariada de Spearman entre los mismos. Con ello, se ha comprobado que existen correlaciones de interés y que son significativas (al nivel 0,01), de modo bilateral, en algunos casos. En este sentido, aparece una correlación de interés entre una práctica dirigida a estar sano y en forma, con la predisposición hacia la misma para mantener la línea y tener buen aspecto físico (el ítem 1 con ítem $4: \mathrm{r}=.52 ; p=.00$ ). Destacamos la dependencia de la práctica de la actividad física por ser una práctica común de los amigos, se propicia el encuentro con ellos y se obtiene otros nuevos (el ítem 5 con el ítem $7: \mathrm{r}=.49 ; p=.00$ ). Además, se da una correlación positiva entre el gusto por la práctica de actividad físico-deportiva y el hecho de que la misma pueda resultar divertida (el ítem 6 con el ítem 10: r=.40; $p=.00$ ). De igual forma, entre la realización de la práctica físico-deportiva de familiares cercanos y la práctica para agradar a los padres (el ítem 8 con el ítem 9: r=.40; $p=.00$ ).

\section{Práctica de la actividad físico-deportiva}

Cuando se les pregunta a la población estudiada si practican actividad físicodeportiva, obtenemos que el $49.58 \%$ contestan afirmativamente, tal y como podemos apreciar en la Tabla 2. Sobre ésta respuesta nos encontramos diferencias en función del género, donde el 70.78\% de los chicos practican uno o varios deportes frente a las chicas que tan sólo practican la actividad un $32.08 \%$. La prueba chi-cuadrado, nos indica que estas diferencias son significativas $\left(\chi^{2}=144.821 ; p=.00\right)$. 
Tabla 2. Práctica de la actividad físico-deportiva

\begin{tabular}{cccccccccc}
\hline & \multicolumn{3}{c}{$\begin{array}{c}\text { Chicos } \\
(\mathrm{n}=389)\end{array}$} & \multicolumn{3}{c}{$\begin{array}{c}\text { Chicas } \\
(\mathrm{n}=468)\end{array}$} & \multicolumn{3}{c}{$\begin{array}{c}\text { Total } \\
(\mathrm{n}=857)\end{array}$} \\
\cline { 2 - 11 } & $N$ & $\%$ & $\begin{array}{c}\% \\
\text { válido }\end{array}$ & $N$ & $\%$ & $\%$ & $N$ & $\%$ & $\%$ \\
& & & & & válido & & & válido \\
\hline No practica actividad física & 109 & $27.90 \%$ & $29.22 \%$ & 307 & $65.50 \%$ & $67.92 \%$ & 416 & $48.54 \%$ & $50.42 \%$ \\
Práctica actividad física & 264 & $67.80 \%$ & $70.78 \%$ & 145 & $30.90 \%$ & $32.08 \%$ & 409 & $47.72 \%$ & $49.58 \%$ \\
Perdidos sistema & 16 & $4.11 \%$ & & 16 & $3.40 \%$ & & 32 & $3.73 \%$ & \\
\hline Total & 389 & $99.8 \%$ & $100 \%$ & 468 & $99.80 \%$ & $100 \%$ & 857 & $100 \%$ & $100 \%$ \\
\hline
\end{tabular}

\section{Motivos de práctica de la actividad físico-deportiva}

De los 409 estudiantes de secundaria que manifiestan que efectúan actividad físicodeportiva, se exponen los motivos que les lleva a esta práctica a partir de las medidas de tendencia central. Atendiendo a los resultados expuestos en la Tabla 3, podemos apreciar que las medias de cada uno de los sectores del colectivo estudiado oscilan entre 1.52 (Ítem 9) a 3.60 (Ítem 6) en los chicos y de 1.42 (Ítem 9) a 3.59 (Ítem 1) en las chicas. En base a ello, podemos decir que en la mayoría de los casos se efectúan valoraciones positivas con respecto a los ítems específicos, situándolos por encima del punto central de la escala (que en esta escala sería el 2).

Tabla 3. Medidas de tendencia central: media y desviación típica

\begin{tabular}{|c|c|c|c|c|c|c|}
\hline \multirow{3}{*}{ Ítems } & \multicolumn{6}{|c|}{ Colectivo } \\
\hline & \multicolumn{2}{|c|}{$\begin{array}{l}\text { Chicos } \\
(\mathrm{n}=264)\end{array}$} & \multicolumn{2}{|c|}{$\begin{array}{l}\text { Chicas } \\
(\mathrm{n}=145)\end{array}$} & \multicolumn{2}{|c|}{$\begin{array}{c}\text { Total } \\
(\mathrm{n}=409)\end{array}$} \\
\hline & $M$ & $D T$ & $M$ & $D T$ & $M$ & $D T$ \\
\hline Ítem 1. Por estar sano y en forma. & 3.50 & $(.69)$ & 3.59 & $(.59)$ & 3.53 & $(.66)$ \\
\hline Ítem 2. Porque me gusta competir. & 2.88 & $(.93)$ & 2.13 & $(.89)$ & 2.62 & $(.98)$ \\
\hline Ítem 3. Porque quiero hacer carrera deportiva. & 2.10 & $(.95)$ & 1.85 & $(.92)$ & 2.01 & $(.95)$ \\
\hline $\begin{array}{l}\text { Ítem 4. Para mantener la línea y tener buen aspecto } \\
\text { físico. }\end{array}$ & 3.04 & $(.84)$ & 3.19 & $(.73)$ & 3.09 & $(.81)$ \\
\hline $\begin{array}{l}\text { Ítem 5. Para encontrarme con amigos y hacer otros } \\
\text { nuevos. }\end{array}$ & 2.95 & $(.86)$ & 2.81 & $(.86)$ & 2.90 & $(.86)$ \\
\hline Ítem 6. Porque me gusta hacer deporte. & 3.60 & $(.61)$ & 3.38 & $(.67)$ & 3.52 & $(.64)$ \\
\hline Ítem 7. Porque mis mejores amigos también practican. & 2.49 & $(.89)$ & 2.09 & $(.83)$ & 2.35 & $(.89)$ \\
\hline $\begin{array}{l}\text { Ítem } 8 \text {. Porque mis familiares cercanos también prac- } \\
\text { tican. }\end{array}$ & 2.03 & $(.83)$ & 1.94 & $(.75)$ & 2.00 & $(.81)$ \\
\hline Ítem 9. Para agradar a mis padres. & 1.52 & $(.73)$ & 1.42 & $(.57)$ & 1.48 & $(.68)$ \\
\hline $\begin{array}{l}\text { Ítem 10. Porque me divierte para ocupar el tiempo } \\
\text { libre. }\end{array}$ & 3.30 & $(.78)$ & 3.33 & $(.66)$ & 3.31 & $(.74)$ \\
\hline
\end{tabular}


Lo más valorado de las poblaciones estudiadas son los aspectos del ítem 1, el ítem 6 y el ítem 10. El ítem 1, "Por estar sano y en forma" (3.53), el ítem 6 "Porque me gusta hacer deporte" (3.52) y el ítem 10 "Porque me divierte para ocupar el tiempo libre" (3.31). Los componentes menos valorados son los contemplados en el ítem 9, ítem 8 y el ítem 3. El ítem 9, "Para agradar a mis padres" (1.48). El ítem 8, "Porque mis familiares cercanos también practican” (2.00) y el ítem 3, "Porque quiero hacer carrera deportiva” (2.01).

Para apreciar las diferencias entre el género (tabla 4), aplicando la Prueba de MannWhitney, se evidencian diferencias significativas $(p<.05)$ en la percepción de chicos y chicas respecto a los ítems $2,3,6$ y 7 .

Tabla 4. Estadísticos de contraste mediante la variable de agrupación sexo

\begin{tabular}{ccccc}
\hline Ítems & U de Mann-Whitney & W de Wilcoxon & $\mathrm{Z}$ & $\mathrm{p}$ \\
\hline Ítem 1 & 17642.00 & 52622.00 & -1.14 & .26 \\
Ítem 2 & 10641.00 & 20652.00 & -7.36 & .00 \\
Ítem 3 & 15573.50 & 25584.50 & -2.75 & .00 \\
Ítem 4 & 16933.500 & 51386.50 & -1.60 & .11 \\
Ítem 5 & 17180.00 & 27333.00 & -1.49 & .14 \\
Ítem 6 & 15321.50 & 25474.50 & -3.57 & .00 \\
Ítem 7 & 14084.00 & 24237.00 & -4.31 & .00 \\
Ítem 8 & 17874.00 & 28027.00 & -.84 & .40 \\
Ítem 9 & 17912.500 & 28065.50 & -.92 & .35 \\
Ítem 10 & 18569.00 & 28722.00 & -.17 & .86 \\
\hline
\end{tabular}

Los chicos valoran más que las chicas, la "Práctica por gusto hacia la competición" $(\mathrm{z}=-7.36 ; \mathrm{p}=.00)$ (Ítem 2), "Porque quiero hacer carrera deportiva” ( $\mathrm{z}=-2.75 ; p=.00)$ (Ítem 3), la "Práctica por gusto hacia el deporte" ( $\mathrm{z}=-3.57$; $p=.00$ ) (Ítem 6) y la "Práctica porque mis mejores amigos también practican” ( $\mathrm{z}=-4.31 ; p=.00)$ (Ítem 7).

Al intentar comparar los distintos tipos de centros, conforme al contraste realizado mediante la prueba de Kruskal-Wallis, no se han dado diferencias entre los centros urbanos, periurbanos y rurales (tabla 5). 
Tabla 5. Estadísticos de contraste según la variable de agrupación tipo de centro

\begin{tabular}{|c|c|c|c|c|c|c|c|c|c|}
\hline \multirow[t]{2}{*}{ Ítems } & \multicolumn{2}{|c|}{ Urbano } & \multicolumn{2}{|c|}{ Periurbano } & \multicolumn{2}{|c|}{ Rural } & \multicolumn{3}{|c|}{ Prueba de Kruskal-Wallis } \\
\hline & $M$ & $D T$ & $M$ & $D T$ & $M$ & $D T$ & $\chi^{2}$ & $\mathrm{gl}$ & $\mathrm{p}$ \\
\hline Ítem 1 & 3,56 & $(, 65)$ & 3,48 & $(, 74)$ & 3,55 & $(, 61)$ & ,54 & 2 &, 76 \\
\hline Ítem 2 & 2,69 & $(1,02)$ & 2,65 & $(, 99)$ & 2,55 & $(, 94)$ & 2,01 & 2 & 37 \\
\hline Ítem 3 & 2,02 & $(1,02)$ & 2,08 & $(, 88)$ & 1,99 & $(, 89)$ &, 87 & 2 & 65 \\
\hline Ítem 4 & 3,10 & $(, 812)$ & 3,09 & $(, 93)$ & 3,10 & $(, 72)$ &, 24 & 2 & 89 \\
\hline Ítem 5 & 2,95 & $(, 88)$ & 2,78 & $(, 88)$ & 2,90 & $(, 83)$ & 2,33 & 2 & ,31 \\
\hline Ítem 6 & 3,52 & $(, 66)$ & 3,52 & $(, 70)$ & 3,55 & $(, 56)$ & ,04 & 2 & ,98 \\
\hline Ítem 7 & 2,42 & $(, 91)$ & 2,41 & $(, 85)$ & 2,25 & $(, 91)$ & 2,81 & 2 &, 25 \\
\hline Ítem 8 & 2,03 & $(, 87)$ & 2,12 & $(, 79)$ & 1,92 & $(, 75)$ & 2,92 & 2 & 23 \\
\hline Ítem 9 & 1,49 & $(, 74)$ & 1,57 & $(, 71)$ & 1,45 & $(, 62)$ & 1,72 & 2 &, 42 \\
\hline Ítem 10 & 3,33 & $(, 78)$ & 3,33 & $(, 75)$ & 3,30 & $(, 68)$ & ,87 & 2 & ,64 \\
\hline
\end{tabular}

\section{Discusión y Conclusiones}

El cuestionario Motivos de práctica físico-deportiva en la etapa adolescente se ha aaptado de forma consistentemente a la población española. De esta forma, observamos como los resultados obtenidos del análisis factorial proporcionan tres subescalas diferentes, compuestas de ítems consistentes, tanto conceptual como estadísticamente, con muy buena confiabilidad en todas (entre .687 y .753 de consistencia interna), con un Alfa total de .81. Indicándo que este cuestionario no sólo discrimina los ítems en la población estudiada, además tiene una alta fiabilidad, permitiéndonos emplearlo y hacer un análisis adecuado de los datos. Los factores identificados que constituyen las subescalas definitivas son:

El factor 1: "Actividad físico-deportiva en el tiempo libre con los amigos", son los ítems asociados a la necesidad de conocer el lugar de los amigos como posible elemento fundamental en la escala de valores de estos jóvenes a la hora de realizar actividad físicodeportiva en su tiempo libre y practicar deporte de competición.

El factor 2: “Influencia de padres y familiares sobre la práctica físico-deportiva”, son los ítems asociados a la necesidad de agradar a los padres y las influencias que reciben de otros familiares para practicar deporte y aquellas cuestiones dirigidas a conocer la orientación de los jóvenes hacia una práctica reglada y competitiva poder dedicarse a ello en un futuro.

El factor 3: "Actividad físico-deportiva orientada a la forma física y a la estética corporal", haciendo referencia a las expectativas, motivaciones e intereses, de los jóvenes perte- 
necientes a la sociedad actual, hacia actividades físicamente activas, como las deportivas, para la mejora de la salud y la imagen corporal con fines estéticos.

A través del mismo, el presente estudio ha examinado los motivos de práctica de actividad físico-deportiva en adolescentes granadinos. Como bien apuntaron Castro-Girona, Pieron y González (2006), es necesario conocer los pensamientos, motivaciones e intereses de los chicos y las chicas sobre la actividad físico-deportiva en general. En este estudio se demuestra que en torno al 50\% de la población estudiada realizan práctica de la actividad físicodeportiva. Además, son los chicos los que más práctica de actividad físico-deportiva efectúan (sobre un 70\%), a diferencia de las chicas que, menos de un tercio de la población no practican. Estos datos son coincidentes con otros estudios (Ministerio de Sanidad, 2007; De Hoyo y Sañudo, 2007; García Ferrando, 2005; Moreno, Rodríguez y Gutiérrez, 2003).

De los resultados obtenidos de los adolescentes granadinos sobre los motivos de práctica de la actividad física se destaca: "por estar sano y en forma", "el gusto por practicar el deporte" y "el divertirse ocupando el tiempo libre”. El motivo más valorado "estar sano y en forma", alude al hábito de relacionado con la salud y la eficiencia motriz de la persona. Este resultado es similar a los encontrados por Castillo y Balaguer (2001), Hellín, Moreno y Rodríguez (2004), Pavón, Moreno, Gutiérrez y Sicilia (2004); Palou, Ponseti, Gili, Borras y Vidal (2005). El motivo "porque me gusta practicar deporte" es de interés de la población estudiada. Estos datos también se ponen de manifiesto en las investigaciones de García Ferrando (2005) y De Hoyo y Sañudo Corrales (2007). La elección del motivo de la "diversión" en el tiempo libre es reconocida por los estudiantes granadinos como un tema que les satisface y que les lleva a implicarse en este tipo de práctica. Contrasta con la mayoría de estudios previos, donde se apunta que los principales motivos de participación en actividades físicodeportivas en la etapa adolescente son la diversión y el ocio (Guillén, Weis y Navarro 2005; Palou, Ponseti, Gili, Borras y Vidal, 2005), tanto para los chicos como para las chicas.

Los motivos que provienen del entorno familiar son poco valorados por los adolescentes granadinos, considerando que agradar a los padres y la práctica deportiva que éstos realizan, favorecen y estimulan poco sus prácticas. Estos datos no coinciden con Manolleses et al. (2008), que apuntan que una participación activa de padres y madres, así como del resto de integrantes del entorno familiar pueden ser elementos fundamentales para desarrollar el incremento de hábitos saludables en los jóvenes. 
Al contrastar las diferencias de género entre los diversos motivos de práctica de actividad físico-deportiva, se ha encontrado que las diferencias significativas en cuanto a género incurren en que los chicos conceden más importancia a la competición, el gusto por el deporte y a estar con los amigos que las chicas. En este sentido, estudios como los de Malina (2001) y Otero (2004) corroboran nuestros resultados respecto a las diferencias encontradas en el interés y la motivación en función del sexo del practicante. De tal manera, que los chicos valoran más aspectos afines a las relaciones sociales y la competición, y las chicas aspectos más cercanos al mantenimiento de la línea, la salud y la estética. En cuanto a las diferencias en las valoraciones realizadas por chicos y chicas, se ha podido verificar que éstos muestran una mayor predisposición hacia la práctica deportiva. Sobre esto, destaca lo apuntado por Moreno, Martínez y Alonso (2006) al indicar que el nivel de diversión en la actividad resulta especialmente importante para las chicas debido a la relación existente entre dicha diversión y la participación en actividades físicas, frente a los chicos que les gusta practicar el deporte. Con referencia a la actividad físico-deportiva para pasar el rato con los amigos, se evidencia que las interacciones con los compañeros resultan un elemento enormemente motivador (Haverly y Davidson, 2005), posiblemente relacionado con lo que interpretamos del estudio de Iannotti, Kogan, Janssen y Boyce (2009) como nueva cultura de los adolescentes.

En la búsqueda de las relaciones entre los diversos motivos de práctica de la actividad físico-deportiva, se ha verificado la relación existente entre la realización de actividad físicodeportiva por estar sano y en forma y la práctica para mantener la línea y tener un buen aspecto físico. Esta correspondencia quedaría justificada por el hecho de que, como bien apuntan Van Exel, de Graaf y Brouwer (2006), en la actualidad, los jóvenes parecen cada vez más interesados en su salud futura. Como prueba de ello, destaca el análisis realizado por Thunfors, Collins y Hanlon (2009). Dicho estudio trató de determinar, los factores individuales que pueden influir en los intereses de los adolescentes de acuerdo a diversas conductas de salud y, por extensión, su posible interés en programas que promovieran estilos de vida saludables. En el mismo, se descubrió un elevado interés por parte de las chicas hacia la pérdida de peso y una alimentación sana. Esto podría deberse a su mayor preocupación por la imagen corporal y la estética (Hellín, Moreno y Rodríguez, 2004).

La dependencia positiva de la práctica de la actividad física por ser una práctica común, propiciando el encuentro entre chicos y chicas, obteniendo otros amigos es de gran 
interés para la socialización y mejora del binestar emocional de los jóvenes (Martínez, Inglés, Piqueras y Ramos, 2010). Concuerda con los estudios de Castillo y Balaguer (2001) en la que los chicos valencianos le dan un gran valor a los motivos de estar con los amigos practicando deporte o hacer nuevos amigos mediante el deporte. También De Hoyo y Sañudo (2007) y Ruiz, García y Díaz (2007), hallaron como un motivo muy importante de práctica deportiva por encontrarse con los amigos, a diferencia de Palou, Ponseti, Gili, Borras y Vidal (2005) que no estaban entre los motivos más valorados.

La correlación existente entre el gusto por la práctica deportiva y que ésta pueda ser divertida en los jóvenes, generaría una mayor predisposición y una mayor participación en el futuro. En relación a esto, diversos estudios muestran bajos niveles de actividad físicodeportiva en adolescentes (Michaud, Jeannin y Suris, 2006; Ortega et al., 2005) producidos por una disminución progresiva de la motivación y el interés unida al desarrollo de estos jóvenes (Pavón y Moreno, 2008). Esto justificaría el hecho de que la falta de estímulo sea una de las causas de abandono de la actividad físico-deportiva.

La relación positiva entre la práctica de la actividad físico-deportiva de familiares cercanos y el intentar agradar a los padres mediante este tipo de actividad, supone un foco de atención que no debemos olvidar, ya que la familia es señalada como el soporte fundamental de la práctica juvenil (Escartí, Pascual y Gutierrez, 2005) y algunos modelos de práctica parentales provocan la futura adquisición de hábitos saludables en los jóvenes (Gustafson y Rhodes, 2006; Pearson, Timperio, Salmon, Crawford y Biddle, 2009). Esto coincide con lo apuntado por MacPhail, Collier y O'Sullivan (2009), quienes señalan que en los nuevos estilos de vida de los jóvenes, la familia y los amigos constituyen los pilares básicos para la afiliación de éstos hacia la actividad físico-deportiva de tiempo libre. A pesar de que no existe una concienciación por parte de estos padres y madres sobre la falta de actividad físico-deportiva de sus hijos y como consecuencia de altos índices de sedentarismo y obesidad (Ministerio de Sanidad y Consumo, 2009), sus influencias ayudaría a mejorar de la motivación de sus hijos hacia actividades más activas en su tiempo libre que contribuirían a la salud presente y futura (Amenabar, Sistiaga y García, 2008; Cordente, García y Sillero, 2008; Latorre et al., 2009).

Siguiendo la línea de Castillo y Balaguer (2001), se vuelven a identificar los factores de los motivos de práctica de la actividad física mediante el análisis de los componentes principales. Se ha obtenido tres factores que extraen las ideas fundamentales sobre los motivos 
que tienen los estudiantes granadinos para efectuar este tipo de práctica. Como factor más valorado, se destaca el de actividad físico-deportiva por estar en forma, por una estética y mantenimiento de la línea. Sobre ello, Moreno et al. (2007), señalan una alta preocupación por la imagen corporal en los adolescentes. Es posible que esto sea debido a la influencia del canon estético y de belleza actual, lo que queda justificado en el estudio de Wilson y Rodgers (2002), donde se verificó una relación positiva entre la práctica físico-deportiva y una mejor autopercepción y autoestima física.

A continuación, el factor de la actividad físico-deportiva en el tiempo libre con los amigos es de gran interés para esta población granadina. En el análisis factorial exploratorio que realizaron Moreno, Cervelló y Martínez (2007), le denominaron factor social, que se refería a la práctica de actividad física como medio de establecer, mejorar o mantener las relaciones sociales, en el que incluía los motivos de estar con los amigos, estar con otros que les gustan la misma actividad, conocer a nuevas personas y el gusto de estar con otros haciendo la misma actividad. A este factor, Castillo y Balaguer (2001), le denominaron factor de afiliación, en él contemplaban los motivos que la población valenciana estudiada valoraba para ver a los amigos y hacer nuevos amigos, otorgándole un gran valor.

Se diferencia el valor que los chicos otorgan al factor de actividad físico-deportiva en el tiempo libre y la predisposición hacia las actividades puramente deportivas con respecto a las chicas. Esto nos indica la necesidad de los chicos de probar sus capacidades y habilidades en la competición y de esta manera lo pasan mejor, a diferencia de las chicas que están más preocupadas por la salud y la estética corporal (Hellín, Moreno y Rodríguez, 2004; Moreno, Cervelló y Martínez, 2007; Otero, 2004).

Para concluir, se corroboran algunos de los motivos de práctica señalados por García Ferrando (2005) como más destacados entre esta población: hacer ejercicio físico, diversión y pasar el tiempo, el gusto por el deporte, mantener y/o mejorar la salud y encontrarse con los amigos, como los grandes motivos a tener en cuenta. En nuestro estudio gana peso el factor motivacional relacionado con la actividad físico-deportiva por estar en forma, por una mejor estética y mantenimiento de la línea y el factor motivacional relacionado con la actividad físico-deportiva en el tiempo libre con los amigos. Sobre estos motivos analizados, se deberían tomar decisiones para adoptar estrategias de intervención más cercanas a las expectativas, 
intereses y demandas sociales y juveniles que propicien una mayor participación de estos chicos y chicas, así como la adquisición de estilos de vida más saludables en el futuro.

Como posibles líneas de investigación a desarrollar en el futuro, en la línea de UrichFrench y Cox (2009), Cecchini et al. (2008), Moreno, Alonso, Martínez Galindo y Cervelló, (2005) o Cecchini, González, Carmona y Contreras (2004), resultaría interesante seguir profundizando en el conocimiento de los procesos motivacionales hacia la práctica de actividad físico-deportiva en la etapa adolescente desde la Educación Física. De igual forma, en la línea de Hagger et al. (2009) o Williams, Pocock y Bridge (2009), hacia el desarrollo de posibles modelos motivacionales basados en la influencia de profesores, compañeros y padres, para un aumento en los niveles de actividad físico-deportiva en este grupo de población.

Otra línea de investigación sería el estudio en profundidad y la definición de los perfiles motivacionales en niños y adolescentes, como medio para facilitar a los profesionales de la Educación Física una mayor comprensión de la cultura juvenil para actuar hacia una mejora de la actitud de estos chicos hacia actividades más activas en su tiempo libre. En este sentido, Moreno, Hellín, Hellín, Cervelló y Sicilia (2008), establecieron una clasificación de diferentes perfiles motivacionales en adolescentes respecto a la asignatura de Educación Física que podrían permitir a los profesores de Educación Física poner en práctica estrategias de segmentación con el fin de aumentar la eficacia de sus intervenciones y lograr una participación más activa entre sus alumnos. De igual forma, se ha evidenciado que los alumnos participantes en las clases de Educación Física mostraron tener mayor nivel de optimismo que los no participantes (Abu-Hilal y Zayed, 2011).

\section{Referencias}

Abu-Hilal, M. y Zayed, K. (2011). Optimism and Pessimism of Physical Education and NonPhysical Education Students: Invariance of Structure. Electronic Journal of Research in Educational Psychology, 9(3), 1267-1284.

Amenabar, B., Sistiaga, J. y García, E. (2008). Revisión de los distintos aspectos de la influencia de los padres y las madres en la práctica de la actividad física y el deporte. Apunts, 93, 29-35. 
Angelopoulos, P., Milionis, H., Grammatikaki, E., Moschonis, G. y Manios, Y. (2009). Changes in BMI and blood pressure after a school based intervention: The CHILDREN study. European Journal of Public Health, 19(3), 319-325.

Apovian, C. (2010). The causes, prevalence, and treatment of obesity revisited in 2009: what have we learned so far? American Journal of Clinical Nutrition, 91(1), 277-279.

Ballesteros-Arribas, J., Dal-re Saavedra, M., Pérez-Farinós, N. y Villar-Villalba, C. (2007). La estrategia para la nutrición, actividad física y prevención de la obesidad: estrategia NAOS. Revista Española de Salud Publica, 81, 443-449.

Binkin, N., Fontana, G., Lamberti, A., Cattaneo, C., Baglio, G., Perra, A., et al. (2010). A national survey of the prevalence of childhood overweight and obesity in Italy. Obesity Reviews, 11(1), 2-10.

Castillo, I. y Balaguer, I. (2001). Dimensiones de los motivos de práctica deportiva de los adolescentes valencianos escolarizados. Apunts, 63, 22-29.

Castillo, I. y Molina-García, J. (2009). Adiposity and psychological well-being: effects of physical activity on university students in Valencia, Spain. Revista Panamericana de salud pública, 26(4), 334-340.

Castro-Girona, M., Pieron, M. y González, M.A. (2006). Actitudes y Motivación en Educación Física Escolar. Retos. Nuevas Tendencias en Educación Física, Deporte y Recreación, 10, 5-22.

Cecchini, J., González, C., Carmona, A. y Contreras, O. (2004). Relaciones entre clima motivacional, la orientación de meta, la motivación intrínseca, la autoconfianza, la ansiedad y el estado de ánimo en jóvenes deportistas. Psicothema, 16 (1), 104-109.

Cecchini, J., Gonzalez, C., Méndez, A., Fernández-Río, J., Contreras, O. y Romero, S. (2008). Metas sociales y de logro, persistencia-esfuerzo e intenciones de práctica deportiva en el alumnado de Educación Física. Psicothema, 20 (2), 260-265.

Cecchini, J., Méndez, A. y Muñiz, J. (2002). Motivos de práctica deportiva en escolares españoles. Psicothema, 14, 523-531.

Cervelló, E., Escartí, A. y Guzmán, J. (2007). Youth sport dropout from the achievement goal theory. Psicothema, 19(1), 65-71.

Coelho, R., Sousa, S., Laranjo, M., Monteiro, A., Braganca, G. y Carreiro, H. (2008). Overweight and obesity prevention in the school. Acta Medica Portuguesa, 21(4), 341-344.

Cordente, C., García, P. y Sillero, M. (2008). ¿Tienen los padres parte de responsabilidad en la salud presente y futura de sus hijos adolescentes? Revista Internacional de Derecho y Gestión del Deporte, 1, 36-48. 
De Hoyo, M. y Sañudo, B. (2007). Motivos y hábitos de práctica de actividad física en escolares de 12 a 16 años en una población rural de Sevilla. Revista Internacional de Medicina y Ciencias de la Actividad Física y el Deporte, 7(26), 87-98.

De Meester, F., Van Lenthe, F., Spittaels, H., Lien, N. y De Bourdeaudhuij, I. (2009). Interventions for promoting physical activity among European teenagers: a systematic review. International Journal of behavioral nutrition and physical activity, 6, 82-93.

Eisenmann, J. (2004). Physical activity and cardiovascular disease risk factors in children and adolescents: an overview. Canadian Journal of Cardiology, 20, 295-301.

Ekblom, O., Bak, E. y Ekblom, B. (2009). Trends in body mass in Swedish adolescents between 2001 and 2007. Acta Pediatrica, 98(3), 519-522.

Escartí, A., Pascual, C. y Gutiérrez, M. (2005). Responsabilidad personal y social a través de la educación física y el deporte. Barcelona: Graó.

Gálvez, A., Rodríguez, P.L. y Velandrino, A. (2007). Influencia de determinados motivos de práctica físico-deportiva sobre los niveles de actividad física habitual en adolescentes. Cuadernos de Psicología del Deporte, 7(1), 71-84.

García-Ferrando, M. (2005). Encuesta sobre hábitos deportivos de los españoles: Avance de resultados. Madrid: Centro de Investigaciones Sociológicas.

Guillén, F., Weis, G. y Navarro, M. (2005). Motivos de participación deportiva de niños brasileños atendiendo a sus edades. Apunts, 80, 29-36.

Gustafson, S. y Rhodes, R. (2006). Parental correlates of physical activity in children and early adolescents. Sports Medicine, 36, 79-97.

Hagger, M., Chatzisarantis, N., Hein, V., Soos, I., Karsai, I., Lintunen, T., et al. (2009). Teacher, peer and parent autonomy support in physical education and leisure-time physical activity: A trans-contextual model of motivation in four nations. Psychology and Health, 24(6), 689-711.

Haverly, K. y Davison, K. (2005). Personal fulfillment motivates adolescents to be physically active. Archives of Pediatrics \& Adolescent Medicine, 159, 1115-1120.

Hellín, P., Moreno, J. y Rodríguez, P. (2004). Motivos de práctica físico-deportiva en la región de Murcia. Cuadernos de Psicología del Deporte, 4, 101- 115.

Hong, X., Li, J., Xu, F., Tse, L., Liang, Y., Wang, Z., et al. (2009). Physical activity inversely associated with the presence of depression among urban adolescents in regional China. Bmc Public Health, 9, 148. 
Iannotti, R., Kogan, M., Janssen, I. y Boyce, W. (2009). Patterns of Adolescent Physical Activity, Screen-Based Media Use, and Positive and Negative Health Indicators in the U.S. and Canada. Journal of Adolescent Health, 44, 493-499.

Izquierdo, J., Rodrigo, C., Majem, L., Roman, B. y Aranceta, J. (2009). Obesity in Spain: current scenario and strategies for intervention. BMC Public Health, 9, 148.

Jago, R., McMurray, R., Bassin, S., Pyle, L., Bruecker, S. y Jakicic, J. (2009). Modifying Middle School Physical Education: Piloting Strategies to Increase Physical Activity. Pediatric Exercise Science, 21(2), 171-185.

Judge, L., Petersen, J. y Lydum, M. (2009). The best kept secret in sports. The 2010 Youth Olympic Games. International review for the sociology of sport, 44(2-3), 173-191.

Jurakic, D., Pedisic, Z. y Andrijasevic, M. (2009). Physical Activity of Croatian Population: Cross-sectional Study Using International Physical Activity Questionnaire. Croatian Medical Journal, 50(2), 165-173.

Kijboonchoo, K., Thasanasuwan, W., Seaburin, W., Wimonpeerapattana, W., Srichan y W., Kunapan, P. (2009). Is there any gender difference in physical activity level in thai adolescents? Annals of nutrition and metabolism, 55, 570-579.

Kjonniksen, L., Anderssen, N. y Wold, B. (2009). Organized youth sport as a predictor of physical activity in adulthood. Scandinavian Journal of Medicine and Science In Sports, 19(5), 646-654.

Latorre, P., Gasco, F., García, M., Martínez, R., Quevedo, O., Carmona, F., et al. (2009). Analysis of the influence of the parents in the sports promotion of the children. Journal of Sport and Health Research, 1(1), 12-25.

Lobstein, T., Baur, L. y Uauy, R. (2004). Obesity in children and young people: a crisis in public health. Obesity Reviews, 5(1), 4-85.

Macarro, J., Romero, C. y Torres, J. (2010). Motivos de abandono de la práctica de actividad físico-deportiva en los estudiantes de Bachillerato de la provincia de Granada. Revista de Educación, 353, 495-519.

MacPhail, A., Collier, C. y O’Sullivan, M. (2009). Lifestyles and gendered patterns of leisure and sporting interests among Irish adolescents. Sports Education and Society, 14(3), 281-299.

Malina, R. (2001). Tracking of physical activity across the lifespan. President's council on physical fitness and sports. Research Digest, 3(14), 1-8.

Manonelles Marqueta, P., Alcaraz, J., Álvarez, J., Jiménez, F., Luengo, E., Manuz, B., et al. (2008). The Utility of the Physical Activity and of the Suitable Habits of Nutrition as a 
Mean to Prevent Obesity in Children and Teenagers. Archivos de Medicina del Deporte, 25(5), 333-353.

Martínez, A., Inglés, C., Piqueras, J. y Ramos, V. (2010). Importancia de los amigos y los padres en la salud y el rendimiento escolar. Electronic Journal of Research in Educational Psychology, 8(1), 111-138.

Martínez, A., Romero, C., Delgado, M y Viciana, J. (2010). Construcción y validación del inventario para una escuela activa y saludable (IEASA). Cuadernos de Psicología del Deporte, 10, 63-71

Martínez-Gómez, D., Gómez-Martínez, S., Puertollano, M., Nova, E., Wärnberg, J., Veiga, O., et al. (2009b). Design and evaluation of a treatment programme for Spanish adolescents with overweight and obesity. The EVASYON Study. BMC Public Health, 9, 414-425.

Martínez-Gómez, D., Martínez-De-Haro, V., Del-Campo, J., Zapatera, B., Welk, G., Villagra, A., et al. (2009a). Validez de cuatro cuestionarios para valorar la actividad física en adolescentes españoles. Gaceta Sanitaria, 23(6), 512-517.

Michaud, P., Jeannin, A. y Suris, J. (2006). Correlates of extracurricular sport participation among Swiss adolescents. European Journal of Pediatric, 165(8), 546-555.

Ministerio de Sanidad (2007). Encuesta de Salud. Recuperado el 14 de mayo de 2010, de http://www.msps.es/estadEstudios/estadisticas/encuestaNacional/home.htm.

Ministerio de Sanidad y Consumo (2009). Guía para una escuela activa y saludable: Orientación para los Centros de Educación Primaria. Recuperado el 12 de julio de 2010, de http://www.perseo.aesan.msps.es/docs/docs/guias/escuela_activa.pdf.

Moliner-Urdiales, D., Ruiz, J., Ortega, F., Rey-López, J., Vicente-Rodríguez, G., EspanaRomero, V., et al. (2009). Association of objectively assessed physical activity with total and central body fat in Spanish adolescents, The HELENA Study. International Journal of Obesity, 33(10), 1126-1135.

Morales, P., Urosa, B. y Blanco, A. (2003). Construcción de escalas de actitudes tipo Likert. Madrid: La Muralla.

Moreno, J. A., Rodríguez, P. L., y Gutiérrez, M. (2003). Intereses y actitudes hacia la Educación Física. Revista Española de Educación Física, XI(2), 14-28.

Moreno, J., Alonso, N., Martínez Galindo, C. y Cervelló, E. (2005). Motivación, disciplina, coeducación y estado de flow en educación física: Diferencias según la satisfacción, la práctica deportiva y la frecuencia de práctica. Cuadernos de Psicología del Deporte, 5(1-2), 225-243. 
Moreno, J., Cervelló, E. y Martínez, A. (2007). Validación de la Escala de Medida de los Motivos para la Actividad Física-Revisada en españoles: Diferencias por motivos de participación. Anales de Psicología, 23(1), 167-176.

Moreno, J., González-Cutre, D. y Chillón, M. (2009). Preliminary Validation in Spanish of a Scale Designed to Measure Motivation in Physical Education Classes: The Perceived Locus of Causality (PLOC) Scale. The Spanish Journal of Psychology, 12(1), 327337.

Moreno, J., Hellín, P., Hellín, G., Cervelló, E. y Sicilia, A. (2008). Assessment of Motivation in Spanish Physical Education Students: Applying Achievement Goals and SelfDetermination Theories. The Open Education Journal, 1, 15-22.

Moreno, J., Martínez, C. y Alonso, N. (2006). Actitudes hacia la práctica físico-deportiva según el sexo del practicante. Revista Internacional de Ciencias del Deporte, 3(2), 2043.

Moreno, J., Pavón, A., Gutiérrez, M. y Sicilia, A. (2005). Motivaciones de los universitarios hacia la práctica físico-deportiva. Revista Internacional de Medicina y Ciencias de la Actividad Física y el Deporte, 5(19), 154-165.

Moreno, J., Zomeño, T., Marín, L., Cervello, E. y Ruiz, L. (2009). Variables motivacionales relacionadas con la práctica deportiva extraescolar en estudiantes adolescentes de educación física. Apunts, 95, 38-43.

Nuviala, A., Cruces, A., Martínez, A., Schonemann, O., Abad, J. y Pons, A. (2009). Health self-perception, lifestyle and organized physical activity. Revista Internacional de Medicina y Ciencias de la actividad física y del deporte, 9(36), 414-430.

OMS (2004). Estrategia mundial sobre régimen alimentario, actividad física y salud. Recuperado el 15 de abril de 2009, de http://www.who.int/dietphysicalactivity/strategy/eb11344/strategy_spanish_web.pdf.

Ortega, F., Ruiz, J., Castillo, M., Moreno, L., González-Gross, M., Wärnberg, J., et al. (2005). Bajo nivel de forma física en los adolescentes españoles. Importancia para la salud cardiovascular futura (Estudio AVENA). Revista Española de Cardiología, 58, 898909.

Otero, J. (2004). Hábitos y actitudes de los andaluces ante el deporte (2002). Cádiz: Consejería de Turismo y Deporte. Observatorio del Deporte Andaluz.

Palou, P., Ponseti, X., Gili, M., Borras, P. y Vidal, J. (2005). Motivos para el inicio, mantenimiento y abandono de la práctica deportiva de los preadolescentes de la isla de Mallorca. Apunts, 3, 5-11. 
Parfitt, G., Pavev, T. y Rowlands, A. (2009). Children's physical activity and psychological health: the relevance of intensity. Acta Pediatrica, 98(6), 1037-1043.

Pavón, A. y Moreno, J. (2008). Actitud de los universitarios ante la práctica físico-deportiva: diferencias por géneros. Revista de Psicología del Deporte, 17(1), 7-23.

Pavón, A., Moreno, J., Gutiérrez, M. y Sicilia, A. (2004). Motivos de práctica físico-deportiva según la edad y el género en una muestra de universitarios. Apunts, 76, 13-21.

Pearson, N., Timperio, A., Salmon, J., Crawford, D. y Biddle, S. (2009). Family influences on children's physical activity and fruit and vegetable consumption. International Journal of behavioral nutrition and physical activity, 6, 34-41.

Prista, A., Nhantumbo, L., Saranga, S., Lopes, V., Maia, J., Seabra, A., et al. (2009). Physical Activity Assessed by Accelerometry in Rural African School-age Children and Adolescents. Pediatric Exercise Science, 21(4), 384-399.

Quevedo-Blasco, V., Quevedo-Blasco, R. y Bermúdez, M. (2009). Análisis de la motivación en la práctica de actividad físico-deportiva en adolescentes. Revista de Investigación en Educación, 6, 33-42.

Roman, B., Serra-Majem, L., Pérez-Rodrigo, C., Drobnic, F. y Segura, R. (2009). Physical activity in children and youth in Spain: future actions for obesity prevention. Nutrition Reviews, 67(5), 94-98.

Ruiz, F., García, E. y Díaz, A. (2007). Análisis de las motivaciones de práctica de actividad física y de abandono deportivo en la Ciudad de La Habana (Cuba). Anales de Psicología, 23(1), 152-166.

Shen, B., McCaughtry, N., Martin, J. y Fahlman, M. (2009). Motivational Profiles and Their Associations With Achievement Outcomes. Journal of Teaching Physical Education, 28(4), 441-460.

Thompson, A., McHugh, T., Blanchard, C., Campagna, P., Durant, M., Rehman, L., et al. (2009). Physical activity of children and youth in Nova Scotia from 2001/02 and 2005/06. Preventive Medicine, 49(5), 407-409.

Thunfors, P., Collins, B. y Hanlon, A. (2009). Health behavior interests of adolescents with unhealthy diet and exercise: implications for weight management. Health Education Research, 24(4), 634-645.

Tounian, P. (2009). Childhood obesity: a new vision. Bulletin del academie nationale de medicine, 193(6), 1243-1254.

Trost, S. y Loprinzi, P. (2008). Exercise-Promoting healthy lifestyles in children and adolescents. Journal of Clinical Lipidology, 2(3), 162-168. 
Urich-French, S. y Cox, A. (2009). Using Cluster Analysis to Examine the Combinations of Motivation Regulations of Physical Education Students. Journal of Sport and Exercise Psychology, 31(3), 358-379.

Van Exel, N., de Graaf, G. y Brouwer, W. (2006). Everyone dies, so you might as well have fun! Attitudes of Dutch youths about their health lifestyle. Social Science and Medicine, 63, 2628-2639.

Vuorela, N., Saha, M. y Salo, M. (2009). Prevalence of overweight and obesity in 5-and 12year-old Finnish children in 1986 and 2006. Acta Pediatrica, 98(3), 507-512.

Williams, P., Pocock, B. y Bridge, K. (2009). Kids' lives in adult space and time: How home, community, school and adult work affect opportunity for teenagers in suburban Australia. Health Sociology Review, 18(1), 79-93.

Wilson, P. y Rodgers, W. (2002). The relationship between exercise motives and physical self-esteem in female exercise participants: An application of self-determination theory. Journal of Applied Biobehavioral Research, 7, 30-43.

Woodruff, S., Hanning, R. y Barr, S. (2009). Energy recommendations for normal weight, overweight and obese children and adolescents: are different equations necessary? Obesity reviews, 10(1), 103-108.

Wu, T. (2007). Promoting physical activity among female Taiwanese adolescents. Annals of behavioural medicine, $33,81$.

Yancey, A., Winfield, D., Larsen, J., Anderson, M., Jackson, P., Overton, J., et al. (2009). "Live, Learn and Play": Building strategic alliances between professional sports and public health. Preventive Medicine, 49(4), 322-325. 